\title{
Amanita phalloides poisoning: one harvest, three outcomes
}

\author{
A Pillay Mauree, ${ }^{1}$ T De Maayer, ${ }^{2}$ A Botes, ${ }^{3} \mathrm{~J}$ Fabian, ${ }^{4}$ H Etheredge, ${ }^{5}$ EM Duncan, ${ }^{6} \mathrm{~J}^{\text {Botha }}{ }^{7}$ \\ 1 Medical Officer-Paediatrics- Rahima Moosa Mother and Child Hospital \\ 2 Paediatric gastroenterologist - Rahima Moosa Mother and Child Hospital, Department of Paediatrics and Child Health, \\ School of Clinical Medicine, Faculty of Health Sciences, University of \\ Witwatersrand \\ 3 Lecturer, School of Molecular and Cell Biology, Faculty of Science, University of the Witwatersrand \\ ${ }^{4}$ Wits Donald Gordon Medical Centre, Department of Internal Medicine, School of Clinical Medicine, Faculty of Health \\ Sciences, University of Witwatersrand \\ ${ }^{5}$ Wits Donald Gordon Medical Centre, Wits Department of Internal Medicine, School of Clinical Medicine (Honorary), \\ ${ }^{6}$ Paediatric transplant coordinator, Wits Donald Gordon Medical Centre \\ ${ }^{7}$ Director of Transplantation, Wits Donald Gordon Medical Centre, ${ }^{9}$ Dept. of Surgery, School of Clinical Medicine, Faculty of \\ Health Sciences, University of Witwatersrand
}

Corresponding author: Angidi Pillay Mauree (a121.mauree@gmail.com)

\begin{abstract}
Summary
The case study is to alert all health care workers and the public alike to the dangers of ingesting wild mushrooms. We describe an incidence of Amanita phalloides poisoning in Johannesburg, South Africa, where three different outcomes were experienced. A pregnant mother recovered with conservative management while her unborn foetus died, and her three-yearold daughter required an emergency liver transplant. We outline the clinical course, management options and principles of mushroom identification and toxicology to increase awareness of the presence of Amanita phalloides and its toxic relatives in South Africa. We highlight that early recognition, prompt treatment and referral to a transplant service is life-saving.
\end{abstract}

\section{Case report}

Permission to publish this report was given by the family and the Human Research Ethics Committee (Medical) of the University of the Witwatersrand (Clearance Certificate No: M180487).

\section{Clinical presentation}

A 3-year-7-month-old girl without any known comorbidities was brought to the paediatric emergency department of Rahima Moosa Mother and Child Hospital (RMMCH) in Johannesburg, South Africa, with lethargy and mild dehydration from acute gastroenteritis. On further history, it was found that the girl and her mother had boiled and eaten wild mushrooms obtained from a Johannesburg garden 40 hours prior. They experienced gastrointestinal symptoms (nausea, vomiting, colicky abdominal pain and diarrhea) within 6-10 hours and subsequently presented to their local clinic. Both mother and child deteriorated clinically, and the child was brought to hospital by her aunt while the mother sought medical care at a nearby hospital delivering care to adults.

Initial examination revealed mild dehydration and a lethargic but rousable child. The rest of her examination was unremarkable. Blood results (40 hours post ingestion) showed pre-renal dysfunction (urea $21.2 \mathrm{mmol} / \mathrm{L}$, creatinine $76 \mu \mathrm{mol} / \mathrm{L})$ and markedly elevated liver enzymes: aspartate aminotransferase (AST) $6037 \mathrm{U} / 1$, alanine aminotransferase (ALT) $3318 \mathrm{U} / \mathrm{l}$. The arterial blood gas showed a mild metabolic acidosis with $\mathrm{pH} 7.29, \mathrm{pC}_{2} 26 \mathrm{mmHg}$, bicarbonate $12.2 \mathrm{mmol} / \mathrm{L}$ and base excess -13 with lactate $5.3 \mathrm{mmol} / \mathrm{L}$. Treatment with intravenous fluids was commenced.

Over the next few hours, the patient became more lethargic and her Glasgow Coma Scale (GCS) dropped to 13/15 while hepatomegaly became apparent. The next blood results (48 hours post ingestion) showed similar renal function tests, an International Normalised Ratio (INR) of 7.6 and an activated partial thromboplastin time (aPTT) of 57 seconds (control 26 seconds). Fresh frozen plasma, vitamin K, $\mathrm{N}$-acetylcysteine, lactulose, oral gentamicin and cimetidine were administered to the patient. 
At $60-72$ hours post ingestion, her liver was $8 \mathrm{~cm}$ below the lower costal margin and her GCS had dropped to $11 / 15$. While her renal function was improving (urea $9.4 \mathrm{mmol} / \mathrm{L}$, Creatinine $36 \mu \mathrm{mol} / \mathrm{L}$ ), her liver function tests continued to deteriorate (AST 10613 U/1, ALT 6718 U/1) and INR 6.7.

The following day, at 84-96 hours post ingestion the patient had an upper gastrointestinal tract haemorrhage secondary to her coagulopathy. The patient was transferred to the Wits Donald Gordon Medical Centre (WDGMC) transplant unit. At that time, the renal function was normal, ALT was 11220 U/1, ALT $7962 \mathrm{U} / 1$ and aPTT was $120 \mathrm{~s}$. The patient had a rapid evaluation for liver transplantation that included a liver biopsy which showed extensive hepatocellular necrosis. She was listed for urgent liver transplantation. Forty-eight hours later a blood group compatible deceased donor liver became available and she underwent liver transplant receiving a split liver allograft. The liver was split on the backtable using the ex-situ technique. Implantation was performed by using caval preservation and the 'piggy-back' technique. Recovery after transplant was largely uneventful with liver enzymes returning to normal and rapid resolution of her coagulopathy and encephalopathy. Immunosuppression with Tacrolimus and prednisone was commenced on the first postoperative day and dosing adjusted according to blood levels. The patient developed renal dysfunction that did not require dialysis and renal function had returned to normal by the time she was discharged from hospital on the 13th postoperative day.

The patient's mother, previously completely well, also presented with gastrointestinal symptoms but was discharged home. At 5 days post ingestion, she was diagnosed as having had a miscarriage at 12 weeks and was admitted to the gynaecological ward with jaundice and a GCS of 13/15. Her blood results at that time showed significant renal dysfunction (urea $35 \mathrm{mmol} / \mathrm{L}$, creatinine $219 \mu \mathrm{mol} / \mathrm{L}$ ) and liver dysfunction (ALT $6832 \mathrm{U} / \mathrm{L}$, AST $1431 \mathrm{U} / \mathrm{L}$, INR 4.5, aPTT 53s). She was transferred to the Charlotte Maxeke Johannesburg Academic hospital for further management where she recovered uneventfully.

A subsequent visit to the site where the mushrooms were harvested was made and identical mushrooms were retrieved and submitted for mycological identification.

\section{Mushroom identification}

\section{Materials and Methods:}

A total of four mushroom specimens (WDG1, 2, 3 and 4) were collected and stored at $4^{\circ} \mathrm{C}$ until use. Segments from each mushroom cap were dissected, surfaced sterilised using $70 \%$ $(\mathrm{v} / \mathrm{v})$ ethanol and rinsed twice in sterile distilled water. Sterile segments were plated onto Sabouraud Dextrose Agar (SBA; Biolab) agar ${ }^{1}$ supplemented with $200 \mathrm{mg} / \mathrm{L}$ chloramphenicol (Sigma-Aldrich) and incubated at $22^{\circ} \mathrm{C}$. Fungal cultures were purified by successive inoculation and incubation at $22^{\circ} \mathrm{C}$ on SBA supplemented with $200 \mathrm{mg} / \mathrm{L}$ chloramphenicol.

Based on macro morphological characteristics, isolates WDG1 and WDG4 were selected for molecular identification. Genomic DNA was extracted from fresh cultures using the Quick-DNA ${ }^{\mathrm{TM}}$ Fungal/Bacterial Miniprep Kit (Zymo Research). The internal transcribed spacer (ITS) region was amplified using OneTaq ${ }^{\circledR}$ QuickLoad ${ }^{\circledR} 2 \mathrm{X}$ Master Mix (NEB) with the universal primers ITS1(5'-TCCGTAGGTGAACCTGCGG-3') and ITS4 (5'-TCCTCCGCTTATTGATATGC-3') as previously described. ${ }^{2}$ Amplicons were sequenced in the forward and reverse direction by Inqaba Biotech Pty Ltd (Nimagen, BrilliantDye $^{\mathrm{TM}}$ Terminator Cycle Sequencing Kit V3.1, BRD3-100/1000). Results were obtained by a BLAST search (National Center Biotechnology Information; NCBI).

Sequencing of the ITS region of the rDNA confirmed that both mushroom isolates WDG1 and WDG4 belonged to Amanita phalloides (NCBI Accession numbers: MH458938; MH458939).

\section{Discussion}

Although foraging for wild mushrooms is uncommon in South Africa, it is important for the practitioner to know that Amanita phalloides and its toxic relatives do occur here. ${ }^{3}$ Children or adults may be exposed to these fungi especially in the wet season, and may present with seemingly uncomplicated diseases such as gastroenteritis long before other toxin-induced organ damage manifests.

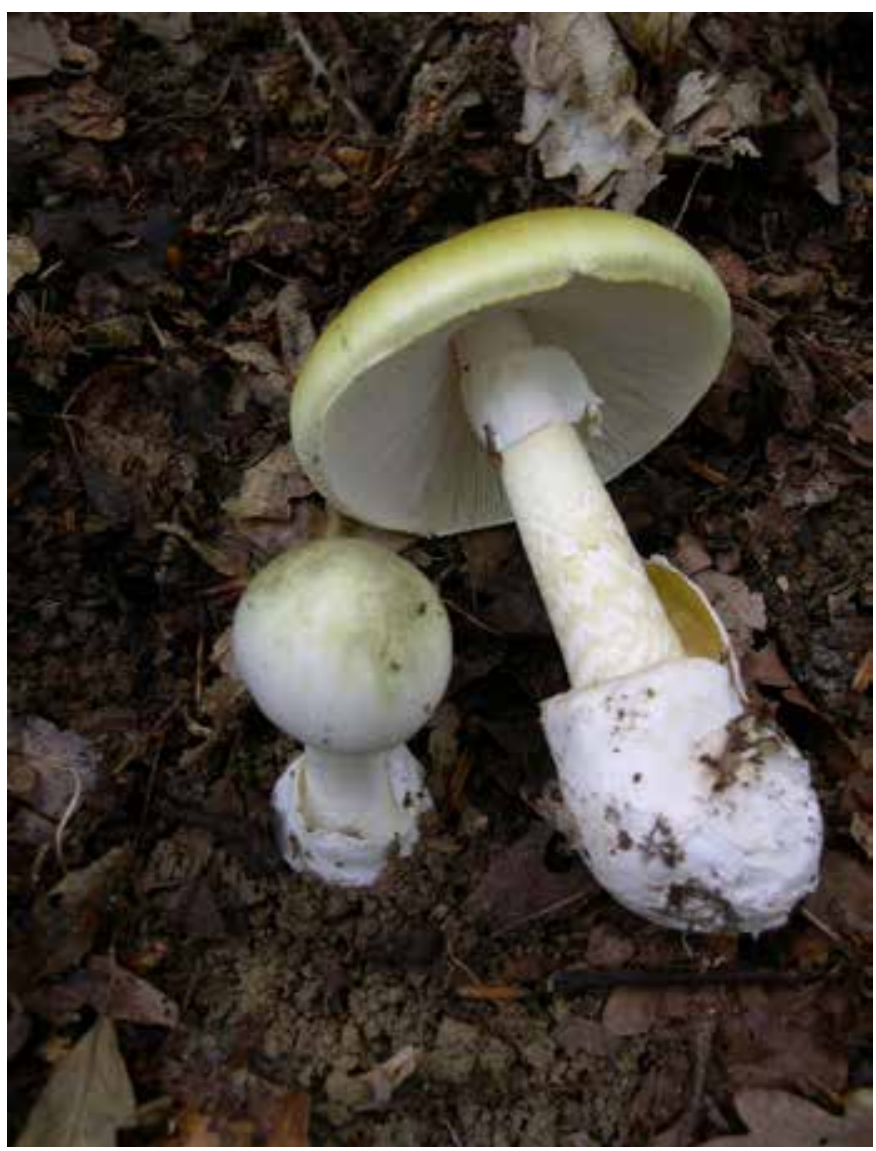

Figure 1: Young Amanita phalloides with a clearly visible saclike volva at the base. By Archenzo - Own work, CC BY-SA 3.0, https://commons. wikimedia.org/w/index.php? curid $=329999$ 
Overall, the Amanita genus is comprised of 500 described species. ${ }^{4}$ The genus is of significance as it contains many poisonous species that can be grouped according to syndromic classification, specifically neurotoxicity, nephrotoxicity and hepatotoxicity. ${ }^{5}$ Hepatotoxic Amanita species, such as $A$. phalloides, are known to contain cyclopetide toxins ${ }^{6}$ (amatoxins and phallotoxins) which are the ultimate cause of liver damage and failure in humans. It is these cyclopeptide toxin-containing species that account for approximately $90 \%$ of fatal mushroom poisonings globally.,

Phalloidin, $\alpha$-amatin and $\beta$-amatin are the major toxins produced by $A$. phalloides. The amatoxins inhibit the synthesis of ribonucleic acid (RNA) leading to cell death, particularly in the liver, with mortality occurring within 2-8 days. ${ }^{6}$ Phallotoxins are considered to have limited role in human fatalities as they are not readily absorbed from the intestine. ${ }^{8,9}$

In South Africa, A. phalloides is regarded as an invasive fungal species. ${ }^{10}$ Originally introduced from Europe and Asia with oak, pine and poplar trees, this ectomycorrhizal fungi (EM) is now widespread throughout South Africa. ${ }^{4,10}$ Despite the species being easily recognizable by the greenish cap and large sac-like volva (Figure 1) at the base, young mushrooms are often misidentified as other edible Amanita spp., resulting in poisoning events. ${ }^{3,4}$ Mortality has been shown to be $>20 \%$ in adults and invariably higher in children $(>50 \%))^{11}$

The usual manifestation post ingestion of $A$. phalloides consists of an asymptomatic period of 8-12 hours followed by gastrointestinal symptoms such as vomiting, abdominal pain and severe secretory diarrhoea in the next 12-24 hours. Non-amatoxin containing mushrooms, which do not affect the liver, usually cause symptoms within 1-2 hours. ${ }^{5}$ In amatoxin poisoning, the patient may present with severe dehydration in the gastroenteritis phase accompanied by hypoglycaemia, oliguria, electrolyte and acid-base imbalances. The diarrhoea usually stops within 24 hours and patient starts feeling better if dehydration has been corrected. The liver and renal function tests may be normal at this stage and the incorrect diagnosis of a simple gastroenteritis can be made.

The liver is usually the main organ affected, with a spectrum of disease from an asymptomatic increase in aminotransferases to acute liver failure with profound coagulopathy and encephalopathy within 4-9 days. ${ }^{5}$ The mortality rate is then $40-80 \%$. Neurotoxicity and renal failure may be present. The severity of $A$. phalloides poisoning depends on the amount of toxin ingested (as little as $0.1 \mathrm{mg} / \mathrm{kg}$ of amatoxin may be lethal) and the time elapsed between consumption and starting of specific treatment., ${ }^{72}$ Since the initial symptoms can be attributed to a simple gastroenteritis episode, there may be significant delay in seeking medical care especially in developing countries where lack of education, poor access to health care and cost are big barriers.

Diagnosis is based on a thorough history and the clinical picture. A comprehensive history entails describing the mushroom, the site from where it was picked, how it was stored or cooked and the onset of symptoms post ingestion. Where available, confirmation of the diagnosis is possible by identification of alpha amanitin in the urine by ELISA. This technique is highly sensitive if done within the first 2 days after ingestion but does not quantify the amount of toxin.? Examination of any remaining mushroom by a mycologist can be invaluable.

There are no standard management guidelines for the child with potential $A$. phalloides ingestion. Most evidence was gathered from experience of clinicians publishing case reports and series.

Early identification and management is critical. The management principles include supporting vital functions, preventing amatoxin absorption, eliminating absorbed amatoxins, using potential antidotes and early involvement of the liver transplant unit. ${ }^{12}$

Preventing amatoxin absorption can be achieved by either gastric lavage or use of activated charcoal. Effectiveness of gastric lavage depends on the time between ingestion and presentation (ideally within 1 hour). Activated charcoal is recommended within the first 60 minutes and it has the added benefit of interrupting the enterohepatic circulation of amatoxins. The recommended charcoal dose is $50 \mathrm{~g}$ every 4 hours. $^{12}$

Out of all the absorbed amatoxins, $60 \%$ is excreted via bile and $40 \%$ renally. Amatoxins can still be found in urine 4 days after ingestion and therefore ensuring adequate diuresis is recommended. Percutaneous biliary drainage has also been shown to help by interrupting the enterohepatic circulation. ${ }^{12}$

Where available, extracorporeal elimination techniques were also found to be useful if started early. Plasmapheresis, haemodialysis and charcoal plasma haemoperfusion, Molecular Adsorbent Recirculating system (MARS) and Fractionated plasma separation and absorption (FPSA) are among the modalities used in amanitin poisoning, with variable success. ${ }^{12,13}$

To try and minimise hepatotoxicity and enhance elimination of amatoxin, many drugs including cimetidine, benzylpenicillin, $\mathrm{N}$-acetylcysteine and silymarin have been suggested as antidotes, with the latter two being the drugs of choice. $^{13}$

$\mathrm{N}$-acetylcysteine has been used for decades and works by scavenging free radicals and as glutathione precursor when endogenous stores are depleted. This antidote may also change amatoxins' intramolecular tryptathione bridge which is vital for toxicity. The usual dose is $150 \mathrm{mg} / \mathrm{kg}$ IV over 15 minutes followed by $50 \mathrm{mg} / \mathrm{kg}$ over 4 hours and then $100 \mathrm{mg} / \mathrm{kg}$ over 16 hours. ${ }^{12}$

Obtained from milk thistle plant Silybum marianum, silymarin has been used for liver disease for a long time. Silybin; the active ingredient of silymarin, has been shown to scavenge free radicals, inhibit lipid peroxidation, increase hepatocyte protein synthesis, decrease the activity of tumor promoters, stabilise mast cells and chelate iron. The overall mortality was $<10 \%$ by using only silymarin as compared to $>20 \%$ with the use of penicillin with or without silymarin. The recommended dose is loading with $5 \mathrm{mg} / \mathrm{kg}$ over 1 hour and then $20-50 \mathrm{mg} / \mathrm{kg} /$ day intravenously and continued for 2-3 days. ${ }^{12,14}$

Failure of medical therapy with severe hepatic dysfunction 
is an indication for emergency liver transplantation. Transplantation criteria are not well described in amatoxin poisoning, unlike in Paracetamol overdose, but a liver transplant remains the only therapeutic method with recognised survival benefit. ${ }^{12}$ A study by Escudié et al. recommends considering liver transplantation for those with an onset of diarrhoea within 8 hours of ingestion, or with an INR $>6$ from day 4 post ingestion, even in the absence of encephalopathy. ${ }^{15}$ These criteria were recently compared to King's College and Ganzert's criteria and found to be $100 \%$ accurate in predicting survival and faster than the other two. ${ }^{16}$

\section{Teaching points}

- Mushroom poisoning is a medical emergency that needs public and health care workers' awareness.

- Prolonged latency to the onset of symptoms from ingestion: suspect Amatoxin intoxication.

- Try to identify the mushroom, consult early and refer early.

- Liver transplant is indicated where conservative management fails.

\section{REFERENCES}

1. Van der Walt JP, Yarrow D. Chapter II - Methods for the isolation, maintenance, classification and identification of yeasts. In: Kreger-van Rij NJWBT-TY. 3rd ed. Amsterdam: Elsevier; 1984. p. 45-104.

2. Innis M, Gelfand DH, Sninsky JJ, White TJ. PCR protocols: a guide to methods and applications [Online]. Vol. 102, Tennessee medicine : Journal of the Tennessee Medical Association. San Diego, California: Academic Press; 1990. p. 29.

3. Steyn DG, Steyn WD, Van der Westhuyzen GCA, Louwrens BA. Mushroom Poisoning. South African Med J. 1953;27(44):47782.

4. Reid D, Eicker A. South African fungi: the genus Amanita. Mycological Research.1991;95(1):80-95. Available at: https:// doi.org/10.1016/S0953-7562(09)81364-6

5. Diaz JH. Syndromic diagnosis and management of confirmed mushroom poisonings. Crit Care Med.
2005;33(2):427-36. Available at: https://doi.org/10.1097/01. CCM.0000153531.69448.49

6. Vetter J. Toxins of Amanita phalloides. Toxicon. 1998;36(1):1324. Available at: https://doi.org/10.1016/S0041-0101(97)000743

7. Berger KJ, Guss DA. Mycotoxins revisited: Part I. J Emerg Med. 2005;28(1):53-62. Available at: https://doi.org/10.1016/j. jemermed.2004.08.013

8. Karlson-Stiber C, Persson H. Cytotoxic fungi--an overview. Toxicon. 2003;42(4):339-49. Available at: https://doi. org/10.1016/S0041-0101(03)00238-1

9. Li C, Oberlies NH. The most widely recognized mushroom: chemistry of the genus Amanita. Life Sci. 2005;78(5):532-8. Available at: https://doi.org/10.1016/j.lfs.2005.09.003

10. Wood AR. Fungi and invasions in South Africa. Bothalia. 2017;47(2):1-16. Available at: https://doi.org/10.4102/abc. v47i2.2124

11. Pawlowska J, Pawlak J, Kaminski A, Jankowska I, Hevelke $\mathrm{P}$, Teisseyre $\mathrm{M}$, et al. Liver transplantation in three family members after Amanita phalloides mushroom poisoning. Transplant Proc. 2002;34(8):3313-4. Available at: https://doi. org/10.1016/S0041-1345(02)03586-8

12. Ye Y, Liu Z. Management of Amanita phalloides poisoning: A literature review and update. J Crit Care. 2018;46:17-22. Available at: https://doi.org/10.1016/j.jcrc.2018.03.028

13. Mas A. Mushrooms, amatoxins and the liver. J Hepatol. 2005;42(2):166-9. Available at: https://doi.org/10.1016/j. jhep.2004.12.003

14. Flora K, Hahn M, Rosen H, Benner K. Milk Thistle (Silybum marianum) for the Therapy of Liver Disease. Am J Gastroenterol. 1998;93(2):139-43. Available at: https://doi. org/10.1111/j.1572-0241.1998.00139.x

15. Escudié L, Francoz C, Vinel J-P, Moucari R, Cournot M, Paradis V, et al. Amanita phalloides poisoning: Reassessment of prognostic factors and indications for emergency liver transplantation. J Hepatol. 2001;46(3):466-73. Available at: https://doi.org/10.1016/j.jhep.2006.10.013

16. Kim YJ, Lee HJ, Ryoo SM, Ahn S, Sohn CH, Seo D-W, et al. Prognostic value of decision criteria for emergency liver transplantation in patients with wild mushroom induced acute liver injury. Hepatobiliary Pancreat Dis Int. 2018;17(3):210-3. Available at: https://doi.org/10.1016/j.hbpd.2018.03.017 